\title{
A New Class of Hermite-Apostol Type Frobenius-Euler Polynomials and Its Applications
}

\author{
Serkan Araci 1,*何, Mumtaz Riyasat ${ }^{2}$, Shahid Ahmad Wani ${ }^{2}$ and Subuhi Khan ${ }^{2}$ \\ 1 Department of Economics, Faculty of Economics, Administrative and Social Sciences, Hasan Kalyoncu \\ University, Gaziantep TR-27410, Turkey \\ 2 Department of Mathematics, Faculty of Science, Aligarh Muslim University, Aligarh 202 002, India; \\ mumtazrst@gmail.com (M.R.); shahidwani177@gmail.com (S.A.W.); subuhi2006@gmail.com (S.K.) \\ * Correspondence: serkan.araci@hku.edu.tr; Tel.: +90-5366750331
}

Received: 6 November 2018; Accepted: 15 November 2018; Published: 19 November 2018

\begin{abstract}
The article is written with the objectives to introduce a multi-variable hybrid class, namely the Hermite-Apostol-type Frobenius-Euler polynomials, and to characterize their properties via different generating function techniques. Several explicit relations involving Hurwitz-Lerch Zeta functions and some summation formulae related to these polynomials are derived. Further, we establish certain symmetry identities involving generalized power sums and Hurwitz-Lerch Zeta functions. An operational view for these polynomials is presented, and corresponding applications are given. The illustrative special cases are also mentioned along with their generating equations.
\end{abstract}

Keywords: Apostol-type Frobenius-Euler polynomials; three-variable Hermite polynomials; symmetric identities; explicit relations; operational connection

MSC: 11B68; 05A10; 11B65

\section{Introduction and Preliminaries}

The multi-variable forms of the special polynomials of mathematical physics help in deriving several useful identities and in introducing new families of special polynomials. We know that the generalized Hermite polynomials are important to deal with quantum mechanical and optical beam transport problems [1] (also see [2,3]). The generating equation for the three-variable Hermite polynomials (3VHP) $H_{n}(x, y, z)[4]$ is given by:

$$
e^{x t+y t^{2}+z t^{3}}=\sum_{n=0}^{\infty} H_{n}(x, y, z) \frac{t^{n}}{n !}
$$

which for $z=0$ reduce to the two-variable Hermite-Kampé de Fériet polynomials (2VHKdFP) $H_{n}(x, y)$ [5] and for $z=0, x=2 x$ and $y=-1$ become the classical Hermite polynomials $H_{n}(x)$ [6].

For $u \in \mathbb{C}, u \neq 1$, the generating equation for the Apostol-type Frobenius-Euler polynomials (ATFEP) $\mathfrak{F}_{n}^{(\alpha)}(x ; u ; \lambda)$, of order $\alpha \in \mathbb{C}$, is given by [7]:

$$
\left(\frac{1-u}{\lambda e^{t}-u}\right)^{\alpha} e^{x t}=\sum_{n=0}^{\infty} \mathfrak{F}_{n}^{(\alpha)}(x ; u ; \lambda) \frac{t^{n}}{n !}
$$

which for $x=0$ gives the Apostol-type Frobenius-Euler numbers (ATFEN) $\mathfrak{F}_{n}^{(\alpha)}(u ; \lambda)$, of order $\alpha$ such that:

$$
\left(\frac{1-u}{\lambda e^{t}-u}\right)^{\alpha}=\sum_{n=0}^{\infty} \mathfrak{F}_{n}^{(\alpha)}(u ; \lambda) \frac{t^{n}}{n !}
$$


For $u=-1$, the ATFEP reduce to the Apostol-Euler polynomials $\mathfrak{E}_{n}^{(\alpha)}(x ; \lambda)$ [8], which for $\lambda=1$, become the Euler polynomials $E_{n}^{(\alpha)}(x)$ [9]. Furthermore, the ATFEP for $\lambda=1$ becomes the Frobenius-Euler polynomials $\mathfrak{F}_{n}^{(\alpha)}(x ; u)$ [10].

The generating equations for the special polynomials are important from different view points and help in finding connection formulas, recursive relations and difference equations and in solving enumeration problems in combinatorics and encoding their solutions.

We intended to introduce a new hybrid class, namely the class of three-variable Hermite-Apostol-type Frobenius-Euler polynomials (3VHATFEP).

Upon replacing the powers $x^{n}$ by the polynomials $H_{n}(x, y, z)$ for $(n=0,1,2, \ldots)$ in Equation (2) and upon the use of Equation (1), we have:

For $u, \lambda \in \mathbb{C}, u \neq 1$, the three-variable Hermite-Apostol-type Frobenius-Euler polynomials ${ }_{H} \mathcal{F}_{n}^{(\alpha)}(x, y, z ; u ; \lambda)$, of order $\alpha \in \mathbb{C}$, are defined by the following generating function:

$$
\left(\frac{1-u}{\lambda e^{t}-u}\right)^{\alpha} e^{x t+y t^{2}+z t^{3}}=\sum_{n=0}^{\infty} H \mathfrak{F}_{n}^{(\alpha)}(x, y, z ; u ; \lambda) \frac{t^{n}}{n !},
$$

which for $\lambda=1$ becomes the three-variable Hermite-Frobenius-Euler polynomials $\mathfrak{F}_{n}^{(\alpha)}(x, y, z ; u)$, of order $\alpha$, which again for $\alpha=1$, give the three-variable Hermite-Frobenius-Euler polynomials $H \mathfrak{F}_{n}(x, y, z ; u)$.

Again, the 3VHATFEP for $u=-1$ give the three-variable Hermite-Apostol-Euler polynomials ${ }_{H} \mathfrak{E}_{n}^{(\alpha)}(x, y, z ; \lambda)$ of order $\alpha$, which for $\lambda=1$ reduce to the three-variable Hermite-Euler polynomials ${ }_{H} E_{n}^{(\alpha)}(x, y, z)$.

The 3VHATFEP are also defined as the discrete Apostol-type Frobenius-Euler convolution of the 3VHP given by:

$$
H \mathfrak{F}_{n}^{(\alpha)}(x, y, z ; u ; \lambda)=n ! \sum_{k=0}^{n} \sum_{r=0}^{[k / 3]} \frac{\mathfrak{F}_{n-k}^{(\alpha)}(u ; \lambda) z^{r} H_{k-3 r}(x, y)}{(n-k) ! r !(k-3 r) !},
$$

where $H_{n}(x, y)$ are the 2VHKdFP.

Next, we deduce certain special cases related to the 3VHATFEP family. Some of these cases are known in the literature. These polynomials are given in Table 1 below.

In this article, the 3VHATFEP are introduced, and certain properties including the explicit relations, summation formulae and symmetric identities for these polynomials are proven using different generating function methods. Some applications for the aforementioned hybrid class of polynomials are given. 
Table 1. Special polynomials related to the ${ }_{H} \mathfrak{F}_{n}^{(\alpha)}(x, y, z ; u ; \lambda)$ family.

\begin{tabular}{|c|c|c|c|}
\hline S.No. & Cases & Name of Polynomial & Generating Function \\
\hline I. & $\begin{array}{l}z=0 \\
z=0, \lambda=1 \\
z=0, \lambda=\alpha=1\end{array}$ & $\begin{array}{l}\text { 2-variable Hermite-Apostol-type Frobenius-Euler polynomials of order } \alpha \\
\text { 2-variable Hermite-Frobenius-Euler polynomials of order } \alpha \\
\text { 2-variable Hermite-Frobenius-Euler polynomials }\end{array}$ & $\begin{array}{l}\left(\frac{1-u}{\lambda e^{t}-u}\right)^{\alpha} e^{x t+y t^{2}}=\sum_{n=0}^{\infty} H \mathfrak{F}_{n}^{(\alpha)}(x, y ; u ; \lambda) \frac{t^{n}}{n !} \\
\left(\frac{1-u}{e^{t}-u}\right)^{\alpha} e^{x t+y t^{2}}=\sum_{n=0}^{\infty} H \mathfrak{F}_{n}^{(\alpha)}(x, y ; u) \frac{t^{n}}{n !} \\
\left(\frac{1-u}{e^{t}-u}\right) e^{x t+y t^{2}}=\sum_{n=0}^{\infty} H \mathfrak{F}_{n}(x, y ; u) \frac{t^{n}}{n !}\end{array}$ \\
\hline II. & $\begin{array}{l}x=2 x, \\
y=-1 ; z=0 \\
x=2 x, y=-1, \\
z=0 ; \lambda=1 \\
x=2 x, y=-1, \\
z=0 ; \alpha=\lambda=1\end{array}$ & $\begin{array}{l}\text { Hermite-Apostol-type Frobenius-Euler polynomials } \\
\text { of order } \alpha \\
\text { Hermite-Frobenius-Euler polynomials } \\
\text { of order } \alpha \\
\text { Hermite-Frobenius-Euler polynomials }\end{array}$ & $\begin{array}{l}\left(\frac{1-u}{\lambda e^{t}-u}\right)^{\alpha} e^{2 x t-t^{2}}=\sum_{n=0}^{\infty} H \mathfrak{F}_{n}^{(\alpha)}(x ; u ; \lambda) \frac{t^{n}}{n !} \\
\left(\frac{1-u}{e^{t}-u}\right)^{\alpha} e^{2 x t-t^{2}}=\sum_{n=0}^{\infty} H \mathfrak{F}_{n}^{(\alpha)}(x ; u) \frac{t^{n}}{n !} \\
\left(\frac{1-u}{e^{t}-u}\right) e^{2 x t-t^{2}}=\sum_{n=0}^{\infty} H \mathfrak{F}_{n}(x ; u) \frac{t^{n}}{n !}\end{array}$ \\
\hline III. & $\begin{array}{l}u=-1 \\
u=-1 \\
\lambda=1 \\
u=-1 \\
\lambda=\alpha=1\end{array}$ & $\begin{array}{l}\text { 3-variable Hermite-Apostol-Euler polynomials of order } \alpha \text { [11] } \\
\text { 3-variable Hermite-Euler polynomials } \\
\text { of order } \alpha \text { [11] } \\
\text { 3-variable Hermite-Euler polynomials [11] }\end{array}$ & $\begin{array}{l}\left(\frac{2}{\lambda e^{t}+1}\right)^{\alpha} e^{x t+y t^{2}+z t^{3}}=\sum_{n=0}^{\infty} H \mathfrak{E}_{n}^{(\alpha)}(x, y, z ; \lambda) \frac{t^{n}}{n !} \\
\left(\frac{2}{e^{t}+1}\right)^{\alpha} e^{x t+y t^{2}+z t^{3}}=\sum_{n=0}^{\infty} H E_{n}^{(\alpha)}(x, y, z) \frac{t^{n}}{n !} \\
\left(\frac{2}{e^{t}+1}\right) e^{x t+y t^{2}+z t^{3}}=\sum_{n=0}^{\infty} H E_{n}(x, y, z) \frac{t^{n}}{n !}\end{array}$ \\
\hline IV. & $\begin{array}{l}u=-1, z=0 \\
u=-1, \lambda=1 \\
z=0 \\
u=-1, \lambda=\alpha=1 \\
z=0\end{array}$ & $\begin{array}{l}\text { 2-variable Hermite-Apostol-Euler polynomials of order } \alpha[11] \\
\text { 2-variable Hermite-Euler polynomials } \\
\text { of order } \alpha \text { [11] } \\
\text { 2-variable Hermite-Euler polynomials [11] }\end{array}$ & $\begin{array}{l}\left(\frac{2}{\lambda e^{t}+1}\right)^{\alpha} e^{x t+y t^{2}}=\sum_{n=0}^{\infty} H \mathfrak{E}_{n}^{(\alpha)}(x, y ; \lambda) \frac{t^{n}}{n !} \\
\left(\frac{2}{e^{t}+1}\right)^{\alpha} e^{x t+y t^{2}}=\sum_{n=0}^{\infty} H E_{n}^{(\alpha)}(x, y) \frac{t^{n}}{n !} \\
\left(\frac{2}{e^{t}+1}\right) e^{x t+y t^{2}}=\sum_{n=0}^{\infty}{ }_{H} E_{n}(x, y) \frac{t^{n}}{n !}\end{array}$ \\
\hline V. & $\begin{array}{l}u=-1, x=2 x \\
y=-1 ; z=0 \\
u=-1, \lambda=1 ; \\
x=2 x, y=-1 ; z=0 \\
u=-1, \lambda=\alpha=1 ; \\
x=2 x, y=-1 ; z=0\end{array}$ & $\begin{array}{l}\text { Hermite-Apostol-Euler } \\
\text { polynomials of order } \alpha \text { [12] } \\
\text { Hermite-Euler polynomials } \\
\text { of order } \alpha \text { [12] } \\
\text { Hermite-Euler polynomials [12] }\end{array}$ & $\begin{array}{l}\left(\frac{2}{\lambda e^{t}+1}\right)^{\alpha} e^{2 x t-t^{2}}=\sum_{n=0}^{\infty} H \mathfrak{E}_{n}^{(\alpha)}(x ; \lambda) \frac{t^{n}}{n !} \\
\left(\frac{2}{e^{t}+1}\right)^{\alpha} e^{2 x t-t^{2}}=\sum_{n=0}^{\infty} H_{n} E_{n}^{(\alpha)}(x) \frac{t^{n}}{n !} \\
\left(\frac{2}{e^{t}+1}\right) e^{2 x t-t^{2}}=\sum_{n=0}^{\infty} H E_{n}(x) \frac{t^{n}}{n !}\end{array}$ \\
\hline
\end{tabular}




\section{Relations}

To derive some relations for the 3VHATFEP, the following results are proven:

Theorem 1. Let $\alpha, \beta \in \mathbb{Z}$, then we have the following relation for the 3 VHATFEP of order $\alpha$ :

$$
H \mathfrak{F}_{n}^{(\alpha \pm \beta)}(x, y, z ; u ; \lambda)=\sum_{k=0}^{n}\left(\begin{array}{l}
n \\
k
\end{array}\right) \mathfrak{F}_{k}^{(\alpha)}(u ; \lambda)_{H} \mathfrak{F}_{n-k}^{( \pm \beta)}(x, y, z ; u ; \lambda) .
$$

Proof. We write the generating Function (4) in the following form:

$$
\sum_{n=0}^{\infty} H \mathfrak{F}_{n}^{(\alpha \pm \beta)}(x, y, z ; u ; \lambda) \frac{t^{n}}{n !}=\left(\frac{1-u}{\lambda e^{t}-u}\right)^{(\alpha \pm \beta)} e^{x t+y t^{2}+z t^{3}}
$$

for which, upon using Equations (3) and (4) and then after simplification, we get Equation (6).

Corollary 1. For $\alpha, \beta \in \mathbb{Z}$, the following relation for the 3 VHAEP of order $\alpha$ holds true:

$$
{ }_{H} \mathfrak{E}_{n}^{(\alpha \pm \beta)}(x, y, z ; \lambda)=\sum_{k=0}^{n}\left(\begin{array}{l}
n \\
k
\end{array}\right) \mathfrak{E}_{k}^{(\alpha)}(\lambda)_{H} \mathfrak{E}_{n-k}^{( \pm \beta)}(x, y, z ; \lambda),
$$

$\mathfrak{E}_{k}^{(\alpha)}(\lambda)$ means Apostol-Euler numbers of order $\alpha$.

Theorem 2. The following recurrence relation for the 3 VHATFEP holds true:

$$
\begin{gathered}
H \mathfrak{F}_{n+1}(x, y, z ; u ; \lambda)=x_{H} \mathfrak{F}_{n}(x, y, z ; u ; \lambda)+2 y n{ }_{H} \mathfrak{F}_{n-1}(x, y, z ; u ; \lambda)+3 z n(n-1) \\
H \mathfrak{F}_{n-2}(x, y, z ; u ; \lambda)-\frac{\lambda}{1-u} \sum_{k=0}^{n}\left(\begin{array}{l}
n \\
k
\end{array}\right)_{H} \mathfrak{F}_{n-k}(x, y, z ; u ; \lambda)_{H} \mathfrak{F}_{k}(1,0,0 ; u ; \lambda) .
\end{gathered}
$$

Proof. Taking $\alpha=1$ and then taking the derivative with respect to $t$ in Equation (4), we find:

$$
\sum_{n=0}^{\infty} H \mathfrak{F}_{n+1}(x, y, z ; u ; \lambda) \frac{t^{n}}{n !}=\left(\frac{1-u}{\lambda e^{t}-u}\right) e^{x t+y t^{2}+z t^{3}}\left(x+2 y t+3 z t^{2}\right)-\frac{(1-u) \lambda e^{t}}{\left(\lambda e^{t}-u\right)^{2}} e^{x t+y t^{2}+z t^{3}},
$$

from which, upon using Equation (4) (for $\alpha=1$ ) and after simplifying the resultant equation, it follows that:

$$
\begin{aligned}
\sum_{n=0}^{\infty} \quad H \mathfrak{F}_{n+1}(x, y, z ; u ; \lambda) \frac{t^{n}}{n !}=x \sum_{n=0}^{\infty} H \mathfrak{F}_{n}(x, y, z ; u ; \lambda) \frac{t^{n}}{n !}+2 y \sum_{n=0}^{\infty} H \mathfrak{F}_{n}(x, y, z ; u ; \lambda) \frac{t^{n+1}}{n !}+3 z \\
\sum_{n=0}^{\infty} H \mathfrak{F}_{n}(x, y, z ; u ; \lambda) \frac{t^{n+2}}{n !}-\frac{\lambda}{1-u} \sum_{n=0}^{\infty} H \mathfrak{F}_{n}(x, y, z ; u ; \lambda) \frac{t^{n}}{n !} \sum_{k=0}^{\infty} H \mathfrak{F}_{k}(1,0,0 ; u ; \lambda) \frac{t^{k}}{k !} .
\end{aligned}
$$

Replacing $n \rightarrow n-1, n-2$ and $n-k$ consecutively in the second, third and last term of the above equation on the r.h.s., it follows that:

$$
\begin{aligned}
\sum_{n=0}^{\infty} \quad H \mathfrak{F}_{n+1}(x, y, z ; u ; \lambda) \frac{t^{n}}{n !}=x \sum_{n=0}^{\infty} H \mathfrak{F}_{n}(x, y, z ; u ; \lambda) \frac{t^{n}}{n !}+2 y \sum_{n=0}^{\infty} H \mathfrak{F}_{n-1}(x, y, z ; u ; \lambda) \frac{t^{n}}{(n-1) !}+3 z \\
\quad \sum_{n=0}^{\infty} H \mathfrak{F}_{n-2}(x, y, z ; u ; \lambda) \frac{t^{n}}{(n-2) !}-\frac{\lambda}{1-u} \sum_{n=0}^{\infty} \sum_{k=0}^{\infty} H \mathfrak{F}_{n-k}(x, y, z ; u ; \lambda)_{H} \mathfrak{F}_{k}(1,0,0 ; u ; \lambda) \frac{t^{n}}{k !(n-k) !}
\end{aligned}
$$

which, upon comparing the coefficients of like powers of $t^{n} / \mathrm{n}$ ! on both sides, gives the recurrence Relation (9). 
Corollary 2. The following recurrence relation for the 3VHAEP holds true:

$$
\begin{aligned}
{ }_{H} \mathfrak{E}_{n+1}(x, y, z ; \lambda) & =x_{H} \mathfrak{E}_{n}(x, y, z ; \lambda)+2 y n_{H} \mathfrak{E}_{n-1}(x, y, z ; \lambda)+3 z n(n-1)_{H} \mathfrak{E}_{n-2}(x, y, z ; \lambda) \\
& -\frac{\lambda}{2} \sum_{k=0}^{n}\left(\begin{array}{l}
n \\
k
\end{array}\right){ }_{H} \mathfrak{E}_{n-k}(x, y, z ; \lambda)_{H} \mathfrak{E}_{k}(1,0,0 ; \lambda) .
\end{aligned}
$$

Theorem 3. For $\gamma>0$, the following relation for the 3VHATFEP of order $\alpha$ holds true:

$$
(1-u)_{H}^{\gamma} \mathfrak{F}_{n}^{(\alpha-\gamma)}(x, y, z ; u ; \lambda)=\sum_{k=0}^{n}\left(\begin{array}{l}
n \\
k
\end{array}\right) H \mathfrak{F}_{n-k}^{(\alpha)}(x, y, z ; u ; \lambda) \sum_{p=0}^{\gamma}\left(\begin{array}{l}
\gamma \\
p
\end{array}\right) \lambda^{p} p^{k}(-u)^{\gamma-p} .
$$

Proof. We write the generating Function (4) in the following form:

$$
\sum_{n=0}^{\infty} H \mathfrak{F}_{n}^{(\alpha-\gamma)}(x, y, z ; u ; \lambda) \frac{t^{n}}{n !}=\left(\frac{1-u}{\lambda e^{t}-u}\right)^{\alpha} e^{x t+y t^{2}+z t^{3}}\left(\lambda e^{t}-u\right)^{\gamma}(1-u)^{-\gamma},
$$

which, upon simplifying and again using Equation (4), gives:

$$
\sum_{n=0}^{\infty} H \mathfrak{F}_{n}^{(\alpha-\gamma)}(x, y, z ; u ; \lambda) \frac{t^{n}}{n !}=(1-u)^{-\gamma} \sum_{n=0}^{\infty} H \mathfrak{F}_{n}^{(\alpha)}(x, y, z ; u ; \lambda) \frac{t^{n}}{n !} \sum_{k=0}^{\infty} \sum_{p=0}^{\gamma}\left(\begin{array}{l}
\gamma \\
p
\end{array}\right) \lambda^{p} p^{k}(-u)^{\gamma-p} \frac{t^{k}}{k !} .
$$

Now, simplifying and then comparing the coefficients of the same powers of $t$ in the resultant equation yield Assertion (13).

Corollary 3. For $\gamma>0$, the following relation for the 3 VHAEP of order $\alpha$ holds true:

$$
2^{\gamma}{ }_{H} \mathfrak{E}_{n}^{(\alpha-\gamma)}(x, y, z ; \lambda)=\sum_{k=0}^{n}\left(\begin{array}{l}
n \\
k
\end{array}\right){ }_{H} \mathfrak{E}_{n-k}^{(\alpha)}(x, y, z ; \lambda) \sum_{p=0}^{\gamma}\left(\begin{array}{l}
\gamma \\
p
\end{array}\right) \lambda^{p} p^{k} .
$$

Theorem 4. For $u, \alpha \in \mathbb{C}, u \neq 1$, there is the following relationship between the 3VHATFEP of order $\alpha$ and the generalized Hurwitz-Lerch Zeta function $(G H L Z F) \Phi_{\mu}(z, s, a)$ :

$$
H \mathfrak{F}_{n}^{(\alpha)}(x, y, z ; u ; \lambda)=\left(\frac{u-1}{u}\right)^{\alpha} \sum_{l=0}^{n}\left(\begin{array}{l}
n \\
l
\end{array}\right) \Phi_{\alpha}\left(\frac{\lambda}{u}, l-n, x\right) H_{l}(0, y, z) .
$$

Proof. We write the generating Function (4) in the following form:

$$
\sum_{n=0}^{\infty} H \mathfrak{F}_{n}^{(\alpha)}(x, y, z ; u ; \lambda) \frac{t^{n}}{n !}=(1-u)^{\alpha}\left(\lambda e^{t}-u\right)^{-\alpha} e^{x t+y t^{2}+z t^{3}},
$$

which, upon simplification, becomes:

$$
\sum_{n=0}^{\infty} H \mathfrak{F}_{n}^{(\alpha)}(x, y, z ; u ; \lambda) \frac{t^{n}}{n !}=(1-u)^{\alpha}(-u)^{-\alpha} \sum_{n=0}^{\infty} \sum_{k=0}^{\infty} \frac{(\alpha)_{k}}{k !}\left(\frac{\lambda}{u}\right)^{k} \frac{(k+x)^{n} t^{n}}{n !} e^{y t^{2}+z t^{3}} .
$$

Using Equation (1) and the following formula for the GHLZF $\Phi_{\mu}(z, s, a)$ [13]:

$$
\Phi_{\mu}(z, s, a)=\sum_{n=0}^{\infty} \frac{(\mu)_{n}}{n !} \frac{z^{n}}{(n+a)^{s}}
$$

and after simplifying the resultant equation yield Relation (17). 
Corollary 4. There is the following relationship between the 3VHAEP of order $\alpha$ and generalized Hurwitz-Lerch Zeta function $\Phi_{\mu}(z, s, a)$ :

$$
{ }_{H} \mathfrak{E}_{n}^{(\alpha)}(x, y, z ; \lambda)=2^{\alpha} \sum_{l=0}^{n}\left(\begin{array}{l}
n \\
l
\end{array}\right) \Phi_{\alpha}(-\lambda, l-n, x) H_{l}(0, y, z) .
$$

Theorem 5. Let $\alpha$ and $\gamma$ be nonnegative integers. There is the following relationship between the numbers $S(n, k, \lambda)$ and the 3 VHATFEP of order $\alpha$ :

$$
\begin{aligned}
& \alpha ! \sum_{l=0}^{n}\left(\begin{array}{c}
n \\
l
\end{array}\right) H \mathfrak{F}_{n-l}^{(\alpha)}(x, y, z ; u ; \lambda) S\left(l, \alpha, \frac{\lambda}{u}\right)=\left(\frac{1-u}{u}\right)^{\alpha} H_{n}(x, y, z), \\
& H \mathfrak{F}_{n}^{(\alpha-\gamma)}(x, y, z ; u ; \lambda)=\gamma !\left(\frac{u}{1-u}\right)^{\gamma} \sum_{l=0}^{n}\left(\begin{array}{c}
n \\
l
\end{array}\right) H \mathfrak{F}_{n-l}^{(\alpha)}(x, y, z ; u ; \lambda) S\left(l, \gamma, \frac{\lambda}{u}\right) .
\end{aligned}
$$

Proof. The generating Equation (4) can be formulated as:

$$
\sum_{n=0}^{\infty}{ }_{H} \mathcal{F}_{n}^{(\alpha)}(x, y, z ; u ; \lambda) \frac{t^{n}}{n !}=(1-u)^{\alpha} \frac{1}{\left(\lambda e^{t}-u\right)^{\alpha}} e^{x t+y t^{2}+z t^{3}}
$$

which, upon rearranging the terms using Equation (1) and the following expansion:

$$
\frac{\left(\lambda e^{t}-1\right)^{k}}{k !}=\sum_{n=0}^{\infty} S(n, k, \lambda) \frac{t^{n}}{n !} .
$$

becomes:

$$
\alpha ! \sum_{n=0}^{\infty} H \mathfrak{F}_{n}^{(\alpha)}(x, y, z ; u ; \lambda) \frac{t^{n}}{n !} \sum_{l=0}^{\infty} S\left(l, \alpha, \frac{\lambda}{u}\right) \frac{t^{l}}{l !}=\left(\frac{1-u}{u}\right)^{\alpha} \sum_{n=0}^{\infty} H_{n}(x, y, z) \frac{t^{n}}{n !} .
$$

which, upon rearranging the summation and then simplifying the resultant equation, yields Relation (22).

Again, we consider the following arrangement of the generating Function (4):

$$
\sum_{n=0}^{\infty} H \mathfrak{F}_{n}^{(\alpha-\gamma)}(x, y, z ; u ; \lambda) \frac{t^{n}}{n !}=\left(\frac{1-u}{\lambda e^{t}-u}\right)^{\alpha} e^{x t+y t^{2}+z t^{3}}\left(\frac{u}{1-u}\right)^{\gamma} \gamma ! \frac{\left(\frac{\lambda}{u} e^{t}-1\right)^{\gamma}}{\gamma !},
$$

which, upon the use of Equations (4) and (25), applying the Cauchy product rule and then canceling the same powers of $t$ in resultant the equation, yields Relation (23).

Corollary 5. There is the following relationship between the numbers $S(n, k, \lambda)$ and the 3 VHAEP of order $\alpha$ :

$$
\begin{aligned}
& \alpha ! \sum_{l=0}^{n}\left(\begin{array}{l}
n \\
l
\end{array}\right)_{H} \mathfrak{E}_{n-l}^{(\alpha)}(x, y, z ; \lambda) S(l, \alpha,-\lambda)=(-2)^{\alpha} H_{n}(x, y, z) . \\
& H \mathfrak{E}_{n}^{(\alpha-\gamma)}(x, y, z ; \lambda)=\gamma !\left(\frac{-1}{2}\right)^{\gamma} \sum_{l=0}^{n}\left(\begin{array}{l}
n \\
l
\end{array}\right)_{H} \mathfrak{E}_{n-l}^{(\alpha)}(x, y, z ; \lambda) S(l, \gamma,-\lambda) .
\end{aligned}
$$

In the next section, we derive some summation formulae for the 3VHATFEP.

\section{Summation Formulae}

In order to prove the summation formulae for the 3VHATFEP ${ }_{H} \mathfrak{F}_{n}^{(\alpha)}(x, y, z ; u ; \lambda)$, we have the following theorems: 
Theorem 6. The following implicit summation formula for the 3VHATFEP of order $\alpha$ holds true:

$$
H \mathfrak{F}_{n}^{(\alpha)}(x+w, y, z ; u ; \lambda)=\sum_{k=0}^{n}\left(\begin{array}{l}
n \\
k
\end{array}\right) H \mathfrak{F}_{k}^{(\alpha)}(x, y, z ; u ; \lambda) w^{n-k} .
$$

Proof. Substituting $x \rightarrow x+w$ in (4), then making use of Equation (4) and with the series expansion of $e^{\text {twt }}$ in the resultant equation, we have:

$$
\sum_{n=0}^{\infty} H \mathfrak{F}_{n}^{(\alpha)}(x+w, y, z ; u ; \lambda) \frac{t^{n}}{n !}=\sum_{n=0}^{\infty} \sum_{k=0}^{\infty} H \mathfrak{F}_{k}^{(\alpha)}(x, y, z ; u ; \lambda) w^{n} \frac{t^{n+k}}{n ! k !}
$$

which, upon simplification, gives Assertion (29).

Corollary 6. For $w=1$ in Equation (29), we have:

$$
H \mathfrak{F}_{n}^{(\alpha)}(x+1, y, z ; u ; \lambda)=\sum_{k=0}^{n}\left(\begin{array}{l}
n \\
k
\end{array}\right) H \mathfrak{F}_{k}^{(\alpha)}(x, y, z ; u ; \lambda) .
$$

Theorem 7. The following implicit summation formula for the 3VHATFEP of order $\alpha$ holds true:

$$
H_{H} \mathfrak{F}_{n}^{(\alpha)}(x+v, y+w, z+r ; u ; \lambda)=\sum_{k=0}^{n}\left(\begin{array}{l}
n \\
k
\end{array}\right) H \mathfrak{F}_{n-k}^{(\alpha)}(x, y, z ; u ; \lambda) H_{k}(v, w, r) .
$$

Proof. Replacing $x \rightarrow x+v, y \rightarrow y+w$ and $z \rightarrow z+r$ in the generating Function (4) and by the help of Equations (1) and (4), we find:

$$
\sum_{n=0}^{\infty} H \mathfrak{F}_{n}^{(\alpha)}(x+v, y+w, z+r ; u ; \lambda) \frac{t^{n}}{n !}=\sum_{n=0}^{\infty} \sum_{k=0}^{\infty} H \mathfrak{F}_{n}^{(\alpha)}(x, y, z ; \lambda ; u) H_{k}(v, w, r) \frac{t^{n+k}}{n ! k !}
$$

which, after simplification, gives Formula (32).

Corollary 7. For $r=0$ in Equation (32), we have:

$$
H \mathfrak{F}_{n}^{(\alpha)}(x+v, y+w, z ; u ; \lambda)=\sum_{k=0}^{n}\left(\begin{array}{l}
n \\
k
\end{array}\right) H \mathfrak{F}_{n-k}^{(\alpha)}(x, y, z ; u ; \lambda) H_{k}(v, w) .
$$

Theorem 8. The following implicit summation formula for the 3VHATFEP of order $\alpha$ holds true:

$$
{ }_{H} \mathfrak{F}_{n+k}^{(\alpha)}(p, y, z ; u ; \lambda)=\sum_{l, m=0}^{n, k}\left(\begin{array}{c}
n \\
l
\end{array}\right)\left(\begin{array}{c}
k \\
m
\end{array}\right)(p-x)^{l+m}{ }_{H} \mathfrak{F}_{n+k-l-m}^{(\alpha)}(x, y, z ; u ; \lambda) .
$$

Proof. Reestablishing $t$ by $t+v$ and after using the following rule:

$$
\sum_{N=0}^{\infty} f(N) \frac{(x+y)^{N}}{N !}=\sum_{l, m=0}^{\infty} f(l+m) \frac{x^{l} y^{m}}{l ! m !}
$$

in Equation (4) and then simplifying the resultant equation, it follows that:

$$
e^{-x(t+v)} \sum_{n, k=0}^{\infty} H \mathfrak{F}_{n+k}^{(\alpha)}(x, y, z ; \lambda ; u) \frac{t^{n} v^{k}}{n ! k !}=\left(\frac{1-u}{\lambda e^{t+v}-u}\right)^{\alpha} e^{y(t+v)^{2}+z(t+v)^{3}} .
$$


Replacing $x$ by $p$ in the above equation, equating the resultant equation to the above equation and then expanding the exponential function give:

$$
\sum_{n, k=0}^{\infty} H \mathfrak{F}_{n+k}^{(\alpha)}(p, y, z ; u ; \lambda) \frac{t^{n} v^{k}}{n ! k !}=\sum_{N=0}^{\infty}(p-x)^{N} \frac{(t+v)^{N}}{N !} \sum_{n, k=0}^{\infty} H \mathfrak{F}_{n+k}^{(\alpha)}(x, y, z ; u ; \lambda) \frac{t^{n} v^{k}}{n ! k !} .
$$

Now, using Formula (36) in the above equation and then replacing $n \rightarrow n-l$ and $k \rightarrow k-m$ in the resultant equation, it follows that:

$$
\sum_{n, k=0}^{\infty} H \mathfrak{F}_{n+k}^{(\alpha)}(p, y, z ; u ; \lambda) \frac{t^{n} v^{k}}{n ! k !}=\sum_{n, k=0}^{\infty} \sum_{l, m=0}^{n, k} \frac{(p-x)^{l+m}}{l ! m !} H \mathfrak{F}_{n+k-l-m}^{(\alpha)}(x, y, z ; u ; \lambda) \frac{t^{n} v^{k}}{(n-l) !(k-m) !},
$$

which gives Formula (35).

Corollary 8. For $n=0$ in Equation (35), we have:

$$
{ }_{H} \mathfrak{F}_{k}^{(\alpha)}(p, y, z ; u ; \lambda)=\sum_{m=0}^{k}\left(\begin{array}{c}
k \\
m
\end{array}\right)(p-x)^{m} \mathfrak{F}_{k-m}^{(\alpha)}(x, y, z ; u ; \lambda)
$$

Corollary 9. Replacing $p$ by $p+x$ and taking $z=0$ in Equation (35), we have:

$$
{ }_{H} \mathfrak{F}_{n+k}^{(\alpha)}(p+x, y ; u ; \lambda)=\sum_{l, m=0}^{n, k}\left(\begin{array}{c}
n \\
l
\end{array}\right)\left(\begin{array}{c}
k \\
m
\end{array}\right) p^{l+m} \mathfrak{F}_{n+k-l-m}^{(\alpha)}(x, y ; u ; \lambda) .
$$

Corollary 10. Replacing $p$ by $p+x$ and taking $y=0 z=0$ in Equation (35), we have:

$$
H \mathfrak{F}_{n+k}^{(\alpha)}(p+x ; u ; \lambda)=\sum_{l, m=0}^{n, k}\left(\begin{array}{c}
n \\
l
\end{array}\right)\left(\begin{array}{c}
k \\
m
\end{array}\right) p^{l+m} \mathfrak{F}_{n+k-l-m}^{(\alpha)}(x ; u ; \lambda) .
$$

Corollary 11. For $p=0$ in Equation (35), we have:

$$
{ }_{H} \mathfrak{F}_{n+k}^{(\alpha)}(y, z ; u ; \lambda)=\sum_{l, m=0}^{n, k}\left(\begin{array}{c}
n \\
l
\end{array}\right)\left(\begin{array}{c}
k \\
m
\end{array}\right)(-x)^{l+m}{ }_{H} \mathfrak{F}_{n+k-l-m}^{(\alpha)}(x, y, z ; u ; \lambda) .
$$

Theorem 9. The following relation for the 3VHATFEP of order $\alpha$ holds true:

$$
H \mathfrak{F}_{n}^{(\alpha)}(x, y, z ; u ; \lambda)=\sum_{k=0}^{\left[\frac{n}{3}\right]} \frac{n !}{(n-3 k) ! k !} H \mathfrak{F}_{n-3 k}^{(\alpha)}(x, y ; u ; \lambda) z^{k}
$$

Proof. Using the equation from Table 1(I), the expansion of $e^{z t^{3}}$ in Equation (4) and then simplifying the resulting equation give:

$$
\sum_{n=0}^{\infty} H \mathfrak{F}_{n}^{(\alpha)}(x, y, z ; u ; \lambda) \frac{t^{n}}{n !}=\sum_{n=0}^{\infty}\left(\sum_{k=0}^{\left[\frac{n}{3}\right]} \frac{n !}{(n-3 k) ! k !} H \mathfrak{F}_{n-3 k}^{(\alpha)}(x, y ; u ; \lambda) z^{k}\right) \frac{t^{n}}{n !} .
$$

After comparing the coefficients of same powers of $t^{n} / n !$ in the above equation, we are led to Relation (44). 
Theorem 10. The following relation for the 3VHATFEP of order $\alpha$ holds true:

$$
H \mathfrak{F}_{n}^{(\alpha)}(x, y, z ; u ; \lambda)=\sum_{k=0}^{n} \sum_{s=0}^{\left[\frac{k}{3}\right]} \frac{n !}{(n-k) !(k-3 s) ! s !} \mathfrak{F}_{n-k}^{(\alpha)}(u ; \lambda) H_{k-3 s}(x, y) z^{s}
$$

Proof. Using Equations (3) and (1) (for $z=0$ ), the expansion of $e^{z t^{3}}$ in Equation (4) and after rearranging the terms, it follows that:

$$
\sum_{n=0}^{\infty} H \mathfrak{F}_{n}^{(\alpha)}(x, y, z ; u ; \lambda) \frac{t^{n}}{n !}=\sum_{n=0}^{\infty} \sum_{k=0}^{n}\left(\begin{array}{l}
n \\
k
\end{array}\right) \mathfrak{F}_{n-k}^{(\alpha)}(u ; \lambda)\left(\sum_{s=0}^{\left[\frac{k}{3}\right]} \frac{k !}{(k-3 s) ! s !} H_{k-3 s}(x, y) z^{s}\right) \frac{t^{n}}{n !} .
$$

Upon canceling the coefficients of like powers of $t$ in Equation (47), we get Assertion (46).

Theorem 11. The following relation for the 3 VHATFEP of order $\alpha$ holds true:

$$
H \mathfrak{F}_{n}^{(\alpha)}(x, y, z ; u ; \lambda)=\sum_{s=0}^{\left[\frac{n}{3}\right]\left[\frac{n-3 s}{2}\right]} \sum_{k=0}^{s !(n-3 s-2 k) ! k !} \mathfrak{F}_{n-3 s-2 k}^{(\alpha)}(x ; u ; \lambda) y^{k} z^{s} .
$$

Proof. With the use of Equation (2), the expansions of $e^{y t^{2}}$ and $e^{z t^{3}}$ in Equation (4) and upon simplifying the resulting equation, we obtain:

$$
\sum_{n=0}^{\infty} H \mathfrak{F}_{n}^{(\alpha)}(x, y, z ; u ; \lambda) \frac{t^{n}}{n !}=\sum_{n=0}^{\infty}\left(\sum_{s=0}^{\left[\frac{n}{3}\right]} \sum_{k=0}^{\left[\frac{n-3 s}{2}\right]} \frac{n !}{s !(n-3 s-2 k) ! k !} \mathfrak{F}_{n-3 s-2 k}^{(\alpha)}(x ; u ; \lambda) y^{k} z^{s}\right) \frac{t^{n}}{n !}
$$

Finally, upon equating the coefficients of the same powers of $t$ in the above equation, Relation (48) is proven.

In the next section, we establish some symmetric identities for the 3VHATFEP.

\section{Symmetric Identities}

The identities for the generalized special functions are useful in electromagnetic processes, combinatorics, numerical analysis, etc. Several types of identities and relations related to Apostol-type polynomials and related polynomials are considered in [14-27]. This provides the motivation to explore symmetry identities for the 3VHATFEP. We recall the following:

For any $\gamma \in \mathbb{R}$ or $\mathbb{C}$, the generalized sum of integer powers $\mathcal{S}_{k}(p ; \gamma)$ is given by:

$$
\frac{\gamma^{p+1} e^{(p+1) t}-1}{\gamma e^{t}-1}=\sum_{k=0}^{\infty} \mathcal{S}_{k}(p ; \gamma) \frac{t^{k}}{k !}
$$

which gives:

$$
\mathcal{S}_{k}(p ; \gamma)=\sum_{l=0}^{k} \gamma^{l} l^{k}
$$

For any $\gamma \in \mathbb{R}$ or $\mathbb{C}$, the multiple power sums $\mathcal{S}_{k}^{(l)}(m ; \gamma)$ are given by:

$$
\left(\frac{1-\gamma^{m} e^{m t}}{1-\gamma e^{t}}\right)^{l}=\frac{1}{\gamma^{l}} \sum_{n=0}^{\infty}\left\{\sum_{p=0}^{n}\left(\begin{array}{l}
n \\
p
\end{array}\right)(-l)^{n-p} \mathcal{S}_{k}^{(l)}(m ; \gamma)\right\} \frac{t^{n}}{n !} .
$$

To prove the symmetry identities for the 3VHATFEP, we have the following theorems: 
Theorem 12. For all integers $c, d>0$ and $n \geq 0, \alpha \geq 1, \lambda, u \in \mathbb{C}$, the following symmetry relation between the $3 V H A T F E P$ of order $\alpha$ and the generalized integer power sums holds true:

$$
\begin{aligned}
& \sum_{k=0}^{n}\left(\begin{array}{l}
n \\
k
\end{array}\right) c^{n-k} \mathfrak{F}_{n-k}^{(\alpha)}\left(d x, d^{2} y, d^{3} z ; \lambda ; u\right) \sum_{l=0}^{k}\left(\begin{array}{l}
k \\
l
\end{array}\right) d^{k} u^{c-1} \mathcal{S}_{l}\left(c-1 ; \frac{\lambda}{u}\right)_{H} \mathfrak{F}_{k-l}^{(\alpha-1)}\left(c X, c^{2} Y, c^{3} Z ; \lambda ; u\right) \\
& =\sum_{k=0}^{n}\left(\begin{array}{l}
n \\
k
\end{array}\right) d^{n-k} u^{d-1} H \mathfrak{F}_{n-k}^{(\alpha)}\left(c x, c^{2} y, c^{3} z ; \lambda ; u\right) \sum_{l=0}^{k}\left(\begin{array}{l}
k \\
l
\end{array}\right) c^{k} \mathcal{S}_{l}\left(d-1 ; \frac{\lambda}{u}\right)_{H} \mathfrak{F}_{k-l}^{(\alpha-1)}\left(d X, d^{2} Y, d^{3} Z ; \lambda ; u\right) .
\end{aligned}
$$

Proof. Let

$$
G(t):=\frac{(1-u)^{2 \alpha-1} e^{c d x t+y(c d t)^{2}+z(c d t)^{3}}\left(\lambda^{c} e^{c d t}-u^{c}\right) e^{c d X t+Y(c d t)^{2}+Z(c d t)^{3}}}{\left(\lambda e^{c t}-u\right)^{\alpha}\left(\lambda e^{d t}-u\right)^{\alpha}},
$$

which, upon rearranging the powers and then using Equations (4) and (50) in the resultant equation, yields:

$$
\begin{aligned}
G(t)= & \left(\sum_{n=0}^{\infty} H \mathfrak{F}_{n}^{(\alpha)}\left(d x, d^{2} y, d^{3} z ; \lambda ; u\right) \frac{(c t)^{n}}{n !}\right)\left(u^{c-1} \sum_{l=0}^{\infty} \mathcal{S}_{l}\left(c-1 ; \frac{\lambda}{u}\right) \frac{(d t)^{l}}{l !}\right) \\
& \times\left(\sum_{k=0}^{\infty} H \mathfrak{F}_{k}^{(\alpha-1)}\left(c X, c^{2} Y, c^{3} Z ; \lambda ; u\right) \frac{(d t)^{k}}{k !}\right) .
\end{aligned}
$$

Upon applying the Cauchy product rule in the above equation, we get:

$$
\begin{aligned}
G(t)= & \sum_{n=0}^{\infty}\left(\sum_{k=0}^{n}\left(\begin{array}{l}
n \\
k
\end{array}\right) c^{n-k} d^{k} u^{c-1} H \mathfrak{F}_{n-k}^{(\alpha)}\left(d x, d^{2} y, d^{3} z ; \lambda ; u\right) \sum_{l=0}^{k}\left(\begin{array}{l}
k \\
l
\end{array}\right) \mathcal{S}_{l}\left(c-1 ; \frac{\lambda}{u}\right)\right. \\
& \left.\times_{H} \mathfrak{F}_{k-l}^{(\alpha-1)}\left(c X, c^{2} Y, c^{3} Z ; \lambda ; u\right)\right) \frac{t^{n}}{n !} .
\end{aligned}
$$

In a similar manner, we obtain:

$$
\begin{aligned}
G(t)= & \sum_{n=0}^{\infty}\left(\sum_{k=0}^{n}\left(\begin{array}{l}
n \\
k
\end{array}\right) d^{n-k} c^{k} u^{d-1} H \mathfrak{F}_{n-k}^{(\alpha)}\left(c x, c^{2} y, c^{3} z ; \lambda ; u\right) \sum_{l=0}^{k}\left(\begin{array}{l}
k \\
l
\end{array}\right) \mathcal{S}_{l}\left(d-1 ; \frac{\lambda}{u}\right)\right. \\
& \left.\times_{H} \mathfrak{F}_{k-l}^{(\alpha-1)}\left(d X, d^{2} Y, d^{3} Z ; \lambda ; u\right)\right) \frac{t^{n}}{n !} .
\end{aligned}
$$

Equating the coefficients of the like powers of $t$ in the r.h.s. of Expansions (55) and (56), we are led to Identity (52).

Theorem 13. For each pair of positive integers $c$, $d$ and for all integers $n \geq 0, \alpha \geq 1, \lambda, u \in \mathbb{C}$, the following symmetry identity for the 3VHATFEP of order $\alpha$ holds true:

$$
\begin{aligned}
& \sum_{k=0}^{n}\left(\begin{array}{l}
n \\
k
\end{array}\right) \sum_{i=0}^{c-1} \sum_{j=0}^{d-1} u^{c+d-2}\left(\frac{\lambda}{u}\right)^{i+j} c^{n-k} d^{k} \widetilde{F}_{k}^{(\alpha)}\left(c X+\frac{c}{d} j, c^{2} Y, c^{3} Z ; \lambda ; u\right) H \widetilde{F}_{n-k}^{(\alpha)}\left(d x+\frac{d}{c} i, d^{2} y, d^{3} z ; \lambda ; u\right) \\
= & \sum_{k=0}^{n}\left(\begin{array}{l}
n \\
k
\end{array}\right) \sum_{i=0}^{d-1} \sum_{j=0}^{c-1} u^{c+d-2}\left(\frac{\lambda}{u}\right)^{i+j} d^{n-k} c^{k} H \widetilde{F}_{k}^{(\alpha)}\left(d X+\frac{d}{c} j, d^{2} Y, d^{3} Z ; \lambda ; u\right) H \widetilde{F}_{n-k}^{(\alpha)}\left(c x+\frac{c}{d} i, c^{2} y, c^{3} z ; \lambda ; u\right) .
\end{aligned}
$$

Proof. Let

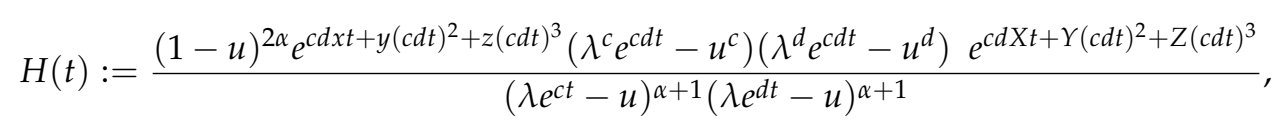


from which, upon rearranging the powers and using the series expansions for $\left(\frac{\lambda^{c} e^{c d t}-u^{c}}{\lambda e^{d t}-u}\right)$ and $\left(\frac{\lambda^{d} e^{c d t}-u^{d}}{\lambda e^{c t} u}\right)$ in the resultant equation, it follows that:

$$
\begin{aligned}
H(t)= & \left(\frac{1-u}{\lambda e^{c t}-u}\right)^{\alpha} e^{d x(c t)+d^{2} y(c t)^{2}+d^{3} z(c t)^{3}} u^{c-1} \sum_{i=0}^{c-1}\left(\frac{\lambda}{u}\right)^{i} e^{d t i} \\
& \times\left(\frac{1-u}{\lambda e^{d t}-u}\right)^{\alpha} e^{c X(d t)+c^{2} Y(d t)^{2}+c^{3} Z(d t)^{3}} u^{d-1} \sum_{j=0}^{d-1}\left(\frac{\lambda}{u}\right)^{j} e^{c t j} .
\end{aligned}
$$

Now, by making use of Equation (4) and the application of the Cauchy product rule in the resultant equation, we have:

$$
\begin{aligned}
H(t)= & \sum_{k=0}^{n}\left(\begin{array}{l}
n \\
k
\end{array}\right) \sum_{i=0}^{c-1} \sum_{j=0}^{d-1} u^{c+d-2}\left(\frac{\lambda}{u}\right)^{i+j} c^{n-k} d^{k} H \mathfrak{F}_{k}^{(\alpha)}\left(c X+\frac{c}{d} j, c^{2} Y, c^{3} Z ; \lambda ; u\right) \\
& H \mathfrak{F}_{n-k}^{(\alpha)}\left(d x+\frac{d}{c} i, d^{2} y, d^{3} z ; \lambda ; u\right) .
\end{aligned}
$$

Following the same lines of proof as above gives another identity:

$$
\begin{aligned}
H(t)= & \sum_{k=0}^{n}\left(\begin{array}{l}
n \\
k
\end{array}\right) \sum_{i=0}^{d-1} \sum_{j=0}^{c-1} u^{d+c-2}\left(\frac{\lambda}{u}\right)^{i+j} d^{n-k} c^{k} H \mathfrak{F}_{k}^{(\alpha)}\left(d X+\frac{d}{c} j, d^{2} Y, d^{3} Z ; \lambda ; u\right) \\
& H \mathfrak{F}_{n-k}^{(\alpha)}\left(c x+\frac{c}{d} i, c^{2} y, c^{3} z ; \lambda ; u\right) .
\end{aligned}
$$

Comparing the coefficients of the same powers of $t$ in the r.h.s. of Expressions (60) and (61) gives Identity (57).

Theorem 14. For each pair of positive integers $c$, $d$ and for all integers $n \geq 0, \alpha \geq 1, \lambda, u \in \mathbb{C}$, the following symmetry identity for the 3 VHATFEP holds true:

$$
\begin{aligned}
\sum_{m=0}^{d-1} u^{d-1}\left(\frac{\lambda}{u}\right)^{m} & \sum_{l=0}^{n}\left(\begin{array}{l}
n \\
l
\end{array}\right)_{H} \mathfrak{F}_{n-l}\left(c x, c^{2} y, c^{3} z ; \lambda ; u\right) d^{n-l}(c m)^{l} \\
= & \sum_{m=0}^{c-1} u^{c-1}\left(\frac{\lambda}{u}\right)^{m} \sum_{l=0}^{n}\left(\begin{array}{l}
n \\
l
\end{array}\right)_{H} \mathfrak{F}_{n-l}\left(d x, d^{2} y, d^{3} z ; \lambda ; u\right) c^{n-l}(d m)^{l} .
\end{aligned}
$$

Proof. Let

$$
N(t):=\frac{(1-u) e^{c d x t+y(c d t)^{2}+z(c d t)^{3}}\left(\lambda^{d} e^{c d t}-u^{d}\right)}{\left(\lambda e^{c t}-u\right)\left(\lambda e^{d t}-u\right)} .
$$

Proceeding on the same lines of proof as in Theorem 13, we get Identity (62). Thus, we omit the proof.

Theorem 15. For each pair of positive integers $c$, $d$ and for all integers $n \geq 0, \alpha \geq 1, \lambda, u \in \mathbb{C}$, the following symmetry relation between the 3 VHATFEP and multiple power sums holds true:

$$
\begin{gathered}
\sum_{l=0}^{n}\left(\begin{array}{c}
n \\
l
\end{array}\right)_{H} \mathfrak{F}_{n-l}\left(d x, d^{2} y, d^{3} z ; \lambda ; u\right) u^{d \alpha} \lambda^{-\alpha} \sum_{m=0}^{l}\left(\begin{array}{c}
l \\
m
\end{array}\right) \sum_{r=0}^{m}\left(\begin{array}{c}
m \\
r
\end{array}\right)(-\alpha)^{m-r} \mathcal{S}_{k}^{(\alpha)}\left(d ; \frac{\lambda}{u}\right) \\
\times \mathfrak{F}_{l-m}^{(\alpha+1)}\left(c X, c^{2} Y, c^{3} Z ; \lambda ; u\right) c^{n-l+m} d^{l-m} \\
=\sum_{l=0}^{n}\left(\begin{array}{c}
n \\
l
\end{array}\right)_{H} \widetilde{F}_{n-l}\left(c x, c^{2} y, c^{3} z ; \lambda ; u\right) u^{c \alpha} \lambda^{-\alpha} \sum_{m=0}^{l}\left(\begin{array}{l}
l \\
m
\end{array}\right) \sum_{r=0}^{m}\left(\begin{array}{c}
m \\
r
\end{array}\right)(-\alpha)^{m-r} \mathcal{S}_{k}^{(\alpha)}\left(c ; \frac{\lambda}{u}\right) \\
\quad \times \mathfrak{F}_{l-m}^{(\alpha+1)}\left(d X, d^{2} Y, d^{3} Z ; \lambda ; u\right) d^{n-l+m} c^{l-m} .
\end{gathered}
$$


Proof. Let:

$$
F(t):=\frac{(1-u)^{\alpha+2} e^{d x(c t)+d^{2} y(c t)^{2}+d^{3} z(c t)^{3}}\left(\lambda^{d} e^{d c t}-u^{d}\right)^{\alpha} e^{c X(d t)+c^{2} Y(d t)^{2}+c^{3} Z(d t)^{3}}}{\left(\lambda e^{d t}-u\right)^{\alpha+1}\left(\lambda e^{c t}-u\right)^{\alpha+1}},
$$

which, upon rearranging the powers and use of Equations (4) and (51) in the resultant equation, yields:

$$
\begin{aligned}
F(t):= & \sum_{n=0}^{\infty} H \widetilde{F}_{n}\left(d x, d^{2} y, d^{3} z ; \lambda ; u\right) c^{n} \frac{t^{n}}{n !} u^{d \alpha} \lambda^{-\alpha} \sum_{m=0}^{\infty} \sum_{r=0}^{m}\left(\begin{array}{c}
m \\
r
\end{array}\right)(-\alpha)^{m-r} \mathcal{S}_{k}^{(\alpha)}\left(d ; \frac{\lambda}{u}\right) c^{m} \frac{t^{m}}{m !} \\
& \sum_{l=0}^{\infty} H \mathfrak{F}_{l}^{(\alpha+1)}\left(c X, c^{2} Y, c^{3} Z ; \lambda ; u\right) d^{l} \frac{l^{l}}{l !} .
\end{aligned}
$$

Now, appropriately applying the using Cauchy product rule in the above equation leads to:

$$
\begin{aligned}
F(t):= & \sum_{n=0}^{\infty} \sum_{l=0}^{n}\left(\begin{array}{l}
n \\
l
\end{array}\right) H \mathfrak{F}_{n-l}\left(d x, d^{2} y, d^{3} z ; \lambda ; u\right) c^{n-l} u^{d \alpha} \lambda^{-\alpha} \sum_{m=0}^{l}\left(\begin{array}{l}
l \\
m
\end{array}\right) \sum_{r=0}^{m}\left(\begin{array}{c}
m \\
r
\end{array}\right)(-\alpha)^{m-r} \mathcal{S}_{k}^{(\alpha)}\left(d ; \frac{\lambda}{u}\right) \\
& H \mathfrak{F}_{l-m}^{(\alpha+1)}\left(c x, c^{2} y, c^{3} z ; \lambda ; u\right) c^{m} d^{l-m} \frac{t^{n}}{n !} .
\end{aligned}
$$

Similarly, we can find:

$$
\begin{aligned}
F(t):= & \sum_{n=0}^{\infty} \sum_{l=0}^{n}\left(\begin{array}{l}
n \\
l
\end{array}\right)_{H} \widetilde{F}_{n-l}\left(c x, c^{2} y, c^{3} z ; \lambda ; u\right) d^{n-l} u^{c \alpha} \lambda^{-\alpha} \sum_{m=0}^{l}\left(\begin{array}{l}
l \\
m
\end{array}\right) \sum_{r=0}^{m}\left(\begin{array}{c}
m \\
r
\end{array}\right)(-\alpha)^{m-r} \mathcal{S}_{k}^{(\alpha)}\left(c ; \frac{\lambda}{u}\right) \\
& H \mathfrak{F}_{l-m}^{(\alpha+1)}\left(d x, d^{2} y, d^{3} z ; \lambda ; u\right) d^{m} c^{l-m} \frac{t^{n}}{n !} .
\end{aligned}
$$

Equating the coefficients of the like powers of $t^{n} / n$ ! in the r.h.s. of Expansions (67) and (68) gives Identity (64).

Theorem 16. For each pair of positive integers $c$, $d$ and for all integers $n \geq 0, \alpha \geq 1, \lambda, u \in \mathbb{C}$, the following symmetry relation between the 3 VHATFEP of order $\alpha$ and multiple power sums holds true:

$$
\begin{aligned}
& \sum_{m=0}^{n}\left(\begin{array}{c}
n \\
m
\end{array}\right)_{H} \mathfrak{F}_{n-m}^{(\alpha)}\left(d x, d^{2} y, d^{3} z ; \lambda ; u\right) c^{n-m} u^{c \alpha} \lambda^{-\alpha} \sum_{r=0}^{m}\left(\begin{array}{c}
m \\
r
\end{array}\right)(-\alpha)^{m-r} \mathcal{S}_{k}^{(\alpha)}\left(c ; \frac{\lambda}{u}\right) d^{m} \\
= & \sum_{m=0}^{n}\left(\begin{array}{c}
n \\
m
\end{array}\right) H \mathfrak{F}_{n-m}^{(\alpha)}\left(c x, c^{2} y, c^{3} z ; \lambda ; u\right) d^{n-m} u^{d \alpha} \lambda^{-\alpha} \sum_{r=0}^{m}\left(\begin{array}{c}
m \\
r
\end{array}\right)(-\alpha)^{m-r} \mathcal{S}_{k}^{(\alpha)}\left(d ; \frac{\lambda}{u}\right) c^{m} .
\end{aligned}
$$

Proof. Let:

$$
M(t):=\frac{(1-u)^{\alpha} e^{d x(c t)+d^{2} y(c t)^{2}+d^{3} z(c t)^{3}}\left(\lambda^{c} e^{c d t}-u^{c}\right)^{\alpha}}{\left(\lambda e^{d t}-u\right)^{\alpha}\left(\lambda e^{c t}-u\right)^{\alpha}} .
$$

Proceeding on the same lines of proof as in Theorem 15, we get Identity (69). Thus, we omit the proof.

Theorem 17. For each pair of positive integers $c$, $d$ and for all integers $n \geq 0, \alpha \geq 1, \lambda, u \in \mathbb{C}$, the following symmetry relation between the 3 VHATFEP of order $\alpha$ and the Hurwitz-Lerch Zeta function holds true:

$$
\begin{aligned}
& \left(\frac{1-u}{u}\right)^{\alpha}(-1)^{\alpha}\left(\sum_{p=0}^{n}\left(\begin{array}{c}
n \\
p
\end{array}\right) \sum_{s=0}^{n-p}\left(\begin{array}{c}
n-p \\
s
\end{array}\right) \Phi_{\alpha}\left(\frac{\lambda}{u}, s-n+p, c x\right) H_{s}\left(0, c^{2} y, c^{3} z\right) d^{n} u^{c} \lambda^{-1}\right. \\
& \left.\sum_{r=0}^{p}\left(\begin{array}{c}
r \\
p
\end{array}\right) \sum_{q=0}^{p-r}\left(\begin{array}{c}
p-r \\
q
\end{array}\right)(-1)^{p-r-q} S_{q}\left(c, \frac{\lambda}{u}\right) H \mathfrak{F}_{r}^{(\alpha)}\left(d X, d^{2} Y, d^{3} Z ; \lambda ; u\right) c^{r} d^{p-r}\right) \\
& =\left(\frac{1-u}{u}\right)^{\alpha}(-1)^{\alpha}\left(\sum_{p=0}^{n}\left(\begin{array}{c}
n \\
p
\end{array}\right) \sum_{s=0}^{n-p}\left(\begin{array}{c}
n-p \\
s
\end{array}\right) \Phi_{\alpha}\left(\frac{\lambda}{u}, s-n+p, c x\right) H_{s}\left(0, d^{2} y, d^{3} z\right) c^{n} u^{d} \lambda^{-1}\right. \\
& \left.\sum_{r=0}^{p}\left(\begin{array}{c}
r \\
p
\end{array}\right) \sum_{q=0}^{p-r}\left(\begin{array}{c}
p-r \\
q
\end{array}\right)(-1)^{p-r-q} S_{q}\left(d, \frac{\lambda}{u}\right) H \mathfrak{F}_{r}^{(\alpha)}\left(c X, c^{2} Y, c^{3} Z ; \lambda ; u\right) d^{r} c^{p-r}\right) .
\end{aligned}
$$


Proof. Let:

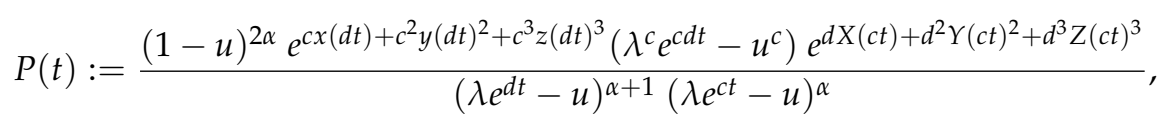

which, upon rearranging the powers and after using Equations (4) and (51) (for $\alpha=1$ ) and the following formula for the generalized binomial theorem:

$$
(1+w)^{-\alpha}=\sum_{m=0}^{\infty}\left(\begin{array}{c}
m+\alpha-1 \\
m
\end{array}\right)(-w)^{m} ; \quad|w|<1,
$$

in the resultant equation becomes:

$$
\begin{aligned}
P(t):= & \left(\frac{1-u}{u}\right)^{\alpha}(-1)^{\alpha} \sum_{m=0}^{\infty}\left(\begin{array}{c}
m+\alpha-1 \\
m
\end{array}\right)\left(\frac{\lambda}{u}\right)^{m} e^{m d t} e^{c x(d t)+c^{2} y(d t)^{2}+c^{3} z(d t)^{3}} u^{c} \lambda^{-1} \sum_{p=0}^{\infty} \sum_{q=0}^{p}\left(\begin{array}{l}
p \\
q
\end{array}\right)(-1)^{p-q} \\
& S_{q}\left(c, \frac{\lambda}{u}\right) d^{p} \frac{t^{p}}{p !} \sum_{r=0}^{\infty} H \widetilde{F}_{r}^{(\alpha)}\left(d X, d^{2} Y, d^{3} Z ; \lambda ; u\right) \frac{(c t)^{r}}{r !} .
\end{aligned}
$$

Simplifying the above equation with the use of Equations (1) and (20) and then using the Cauchy product rule in the resultant equation, we get:

$$
\begin{aligned}
P(t):= & \left(\frac{1-u}{u}\right)^{\alpha}(-1)^{\alpha} \sum_{n=0}^{\infty}\left(\sum_{p=0}^{n}\left(\begin{array}{c}
n \\
p
\end{array}\right) \sum_{s=0}^{n-p}\left(\begin{array}{c}
n-p \\
s
\end{array}\right) \Phi_{\alpha}\left(\frac{\lambda}{u}, s-n+p, c x\right) H_{s}\left(0, c^{2} y, c^{3} z\right) d^{n} u^{c} \lambda^{-1}\right. \\
& \left.\sum_{r=0}^{p}\left(\begin{array}{c}
r \\
p
\end{array}\right) \sum_{q=0}^{p-r}\left(\begin{array}{c}
p-r \\
q
\end{array}\right)(-1)^{p-r-q} S_{q}\left(c, \frac{\lambda}{u}\right) H \mathfrak{F}_{r}^{(\alpha)}\left(d X, d^{2} Y, d^{3} Z ; \lambda ; u\right) c^{r} d^{p-r}\right) \frac{t^{n}}{n !} .
\end{aligned}
$$

In a similar manner, we have:

$$
\begin{aligned}
P(t):= & \left(\frac{1-u}{u}\right)^{\alpha}(-1)^{\alpha} \sum_{n=0}^{\infty}\left(\sum_{p=0}^{n}\left(\begin{array}{c}
n \\
p
\end{array}\right) \sum_{s=0}^{n-p}\left(\begin{array}{c}
n-p \\
s
\end{array}\right) \Phi_{\alpha}\left(\frac{\lambda}{u}, s-n+p, d x\right) H_{s}\left(0, d^{2} y, d^{3} z\right) c^{n} u^{d} \lambda^{-1}\right. \\
& \left.\sum_{r=0}^{p}\left(\begin{array}{c}
r \\
p
\end{array}\right) \sum_{q=0}^{p-r}\left(\begin{array}{c}
p-r \\
q
\end{array}\right)(-1)^{p-r-q} S_{q}\left(d, \frac{\lambda}{u}\right) H \mathfrak{F}_{r}^{(\alpha)}\left(c X, c^{2} Y, c^{3} Z ; \lambda ; u\right) d^{r} c^{p-r}\right) \frac{t^{n}}{n !} .
\end{aligned}
$$

Finally, canceling the coefficients of the same powers of $t$ in the r.h.s. of Expansions (75) and (76), Identity (71) is proven.

Note: The results established above for the 3VHATFEP can be reduced to the illustrative special cases mentioned in Table 1 simply by substituting special values of the variables or parameters. Therefore, we omit them.

\section{Operational Representation}

The classical and Apostol-type Frobenius-Euler numbers and polynomials are the generalization of Euler numbers and polynomials, and these are associated with the Brouwer fixed-point theorem and vector fields [28].

From generating Equation (4), we find that the 3VHATFEP are the solutions of the following equations:

$$
\begin{aligned}
\frac{\partial}{\partial y} H \mathfrak{F}_{n}^{(\alpha)}(x, y, z ; u ; \lambda) & =\frac{\partial^{2}}{\partial x^{2}} H \mathfrak{F}_{n}^{(\alpha)}(x, y, z ; u ; \lambda), \\
\frac{\partial}{\partial z} H \mathfrak{F}_{n}^{(\alpha)}(x, y, z ; u ; \lambda) & =\frac{\partial^{3}}{\partial x^{3}} H \mathfrak{F}_{n}^{(\alpha)}(x, y, z ; u ; \lambda),
\end{aligned}
$$

under the following initial condition:

$$
{ }_{H} \mathfrak{F}_{n}^{(\alpha)}(x, 0,0 ; u ; \lambda)=\mathfrak{F}_{n}^{(\alpha)}(x ; u ; \lambda)
$$


Thus, in view of the above equation, we find that, for the 3VHATFEP, the following operational representation holds true:

$$
H_{\mathfrak{F}_{n}^{(\alpha)}}(x, y, z ; u ; \lambda)=\exp \left(y \frac{\partial^{2}}{\partial x^{2}}+z \frac{\partial^{3}}{\partial x^{3}}\right)\left\{\mathfrak{F}_{n}^{(\alpha)}(x ; u ; \lambda)\right\} .
$$

The operational formalism developed above can be used to obtain the corresponding identities for the 3VHATFEP and for their special cases. To give the applications of the operational representation (80), we apply the operation $\mathcal{O}$ given below:

$\mathcal{O}$ : Operating $\exp \left(y \frac{\partial^{2}}{\partial x^{2}}+z \frac{\partial^{3}}{\partial x^{3}}\right)$ on both sides of a given result.

Consider the following identities for the FEP $\mathfrak{F}_{n}^{(\alpha)}(x ; u)$ from [17]:

$$
\begin{gathered}
u \mathfrak{F}_{n}\left(x ; u^{-1}\right)+\mathfrak{F}_{n}(x ; u)=(1+u) \sum_{k=0}^{n}\left(\begin{array}{l}
n \\
k
\end{array}\right) \mathfrak{F}_{n-k}\left(u^{-1}\right) \mathfrak{F}_{k}(x ; u), \\
\frac{1}{n+1} \mathfrak{F}_{k}(x ; u)+\mathfrak{F}_{n-k}(x ; u)=\sum_{k=0}^{n-1} \frac{\left(\begin{array}{c}
n \\
k
\end{array}\right)}{n-k+1} \sum_{l=k}^{n}\left((-u) \mathfrak{F}_{l-k}(u) \mathfrak{F}_{n-l}(u)+2 u \mathfrak{F}_{n-k}(u)\right) \\
\mathfrak{F}_{k}(x ; u) \mathfrak{F}_{n}(x ; u), \\
\mathfrak{F}_{n}^{(\alpha)}(x ; u)=\sum_{k=0}^{n}\left(\begin{array}{l}
n \\
k
\end{array}\right) \mathfrak{F}_{n-k}^{(\alpha-1)}(u) \mathfrak{F}_{k}(x ; u) \quad\left(n \in \mathbb{Z}_{+}\right), \\
\mathfrak{F}_{n}(x ; u)=\frac{1}{(1-u)^{\alpha}} \sum_{k=0}^{n}\left(\begin{array}{l}
n \\
k
\end{array}\right)\left(\sum_{j=0}^{\alpha}\left(\begin{array}{c}
\alpha \\
j
\end{array}\right)(-u)^{\alpha-j} \mathfrak{F}_{n-k}(j ; u)\right) \mathfrak{F}_{k}^{(\alpha)}(x ; u) \quad\left(n \in \mathbb{Z}_{+}\right),
\end{gathered}
$$

which, upon using operation $(\mathcal{O})$ in both sides, yields the following identities for the polynomials ${ }_{H} \mathfrak{F}_{n}^{(\alpha)}(x, y, z ; u)$ :

$$
\begin{gathered}
u_{H} \mathfrak{F}_{n}\left(x, y, z ; u^{-1}\right)+{ }_{H} \mathfrak{F}_{n}(x, y, z ; u)=(1+u) \sum_{k=0}^{n}\left(\begin{array}{l}
n \\
k
\end{array}\right) \mathfrak{F}_{n-k}\left(u^{-1}\right)_{H} \mathfrak{F}_{k}(x, y, z ; u), \\
\frac{1}{n+1} H \mathfrak{F}_{k}(x, y, z ; u)+{ }_{H} \mathfrak{F}_{n-k}(x, y, z ; u)=\sum_{k=0}^{n-1} \frac{\left(\begin{array}{l}
n \\
k
\end{array}\right)}{n-k+1} \sum_{l=k}^{n}\left((-u) \mathfrak{F}_{l-k}(u) \mathfrak{F}_{n-l}(u)+2 u \mathfrak{F}_{n-k}(u)\right) \\
H \mathfrak{F}_{k}(x, y, z ; u) \mathfrak{F}_{n}(x ; u), \\
H \mathfrak{F}_{n}^{(\alpha)}(x, y, z ; u)=\sum_{k=0}^{n}\left(\begin{array}{l}
n \\
k
\end{array}\right) \mathfrak{F}_{n-k}^{(\alpha-1)}(u)_{H} \mathfrak{F}_{k}(x, y, z ; u) \quad\left(n \in \mathbb{Z}_{+}\right), \\
H \mathfrak{F}_{n}(x, y, z ; u)=\frac{1}{(1-u)^{\alpha}} \sum_{k=0}^{n}\left(\begin{array}{l}
n \\
k
\end{array}\right)\left(\sum_{j=0}^{\alpha}\left(\begin{array}{c}
\alpha \\
j
\end{array}\right)(-u)^{\alpha-j} \mathfrak{F}_{n-k}(j ; u)\right) H \mathfrak{F}_{k}^{(\alpha)}(x, y, z ; u) \quad\left(n \in \mathbb{Z}_{+}\right) .
\end{gathered}
$$

Thus, we find that the aforementioned polynomials, which include the polynomials as their special cases given in Table 1 along with the underlying operational formalism, offer a powerful tool for the investigation of the properties of a wide class of polynomials. Thus, the combination of Hermite and Frobenius-Euler polynomials yields such interesting results.

Further, motivated by the ATFEP $\mathfrak{F}_{n}^{(\alpha)}(x ; u ; \lambda)$, we introduce the Apostol type Frobenius-Genocchi polynomials $\mathfrak{H}_{n}^{(\alpha)}(x ; u ; \lambda)$ (ATFGP). For $u \in \mathbb{C}, u \neq 1$, the ATFGP of order $\alpha \in \mathbb{C}$ are defined by:

$$
\left(\frac{(1-u) t}{\lambda e^{t}-u}\right)^{\alpha} e^{x t}=\sum_{n=0}^{\infty} \mathfrak{H}_{n}^{(\alpha)}(x ; u ; \lambda) \frac{t^{n}}{n !}
$$

which, for $\lambda=\alpha=1$, reduce to the Frobenius-Genocchi polynomials $G_{n}^{F}(x ; u)$ [29]. 
Using the previous approach, we introduce the three-variable Hermite-Apostol-type Frobenius-Genocchi polynomials (3VHATFGP) ${ }_{H} \mathfrak{H}_{n}^{(\alpha)}(x, y, z ; u ; \lambda)$ of order $\alpha \in \mathbb{C}$ defined by:

$$
\left(\frac{(1-u) t}{\lambda e^{t}-u}\right)^{\alpha} e^{x t+y t^{2}+z t^{3}}=\sum_{n=0}^{\infty} H_{\mathfrak{H}_{n}^{(\alpha)}}(x, y, z ; u ; \lambda) \frac{t^{n}}{n !} .
$$

The special members related to the 3VHATFGP $H_{H}^{(\alpha)}(x, y, z ; u ; \lambda)$ can be obtained, and corresponding results for these polynomials and for their special cases can be obtained easily. Thus, we omit them.

\section{Conclusions}

In this paper, a multi-variable hybrid class of the Hermite-Apostol-type Frobenius-Euler polynomials is introduced and their properties are explored using various generating function methods. Several explicit and recurrence relations, summation formulae and symmetry identities are established for these hybrid polynomials. A brief view of the operational approach is also given for these polynomials. The operational representations combined with integral transforms may lead to other interesting results, which may be helpful to the theory of fractional calculus. Several techniques and methods are used in $[30,31]$, which are applicable to the other fields of mathematics. The applicability of these techniques to the hybrid polynomial families can also be explored. These aspects will be undertaken in further investigation.

Author Contributions: All authors contributed equally

Funding: Dr. S. Araci was supported by the Research Fund of Hasan Kalyoncu University in 2018. This work has been done under Post-Doctoral Fellowship (Office Memo No.2/40(38)/2016/R\&D-II/1063) awarded to Dr. M. Riyasat by the National Board of Higher Mathematics, Department of Atomic Energy, Government of India, Mumbai.

Conflicts of Interest: The authors declare no conflict of interest.

\section{References}

1. Dattoli, G.; Lorenzutta, S.; Maino, G.; Torre, A.; Cesarano, C. Generalized Hermite polynomials and super-Gaussian forms. J. Math. Anal. Appl. 1996, 203, 597-609. [CrossRef]

2. Cesarano, C. Operational methods and new identities for Hermite polynomials. Math. Model. Nat. Phenom. 2017, 12, 44-50. [CrossRef]

3. Cesarano, C.; Fornaro, C.; Vázquez, L. A note on a special class of Hermite polynomials. Int. J. Pure Appl. Math. 2015, 98, 261-273. [CrossRef]

4. Dattoli, G. Generalized polynomials operational identities and their applications. J. Comput. Appl. Math. 2000, 118, 111-123. [CrossRef]

5. Appell, P.; de Fériet, J.K. Fonctions Hypergéométriques et Hypersphériques: Polynômes d' Hermite; Gauthier-Villars: Paris, France, 1926.

6. Andrews, L.C. Special Functions for Engineers and Applied Mathematicians; Macmillan Publishing Company: New York, NY, USA, 1985.

7. Özarslan, M.A. Unified Apostol-Bernoulli, Euler and Genocchi polynomials. Comput. Math. Appl. 2011, 62, 2452-2462. [CrossRef]

8. Luo, Q.M. Apostol-Euler polynomials of higher order and the Gaussian hypergeometric function. Taiwan. J. Math. 2006, 10, 917-925. [CrossRef]

9. Erdélyi, A.; Magnus, W.; Oberhettinger, F.; Tricomi, F.G. Higher Transcendental Functions; McGraw-Hill Book Company: New York, NY, USA; Toronto, ON, Canada; London, UK, 1955; Volume III.

10. Carlitz, L. Eulerian numbers and polynomials. Math. Mag. 1959, 32, 247-260. [CrossRef]

11. Khan, S.; Yasmin, G.; Khan, R.; Hassan, N.A.M. Hermite-based Appell polynomials: Properties and applications. J. Math. Anal. Appl. 2009, 351, 756-764. [CrossRef] 
12. Khan, S.; Riyasat, M. A determinantal approach to Sheffer-Appell polynomials via monomiality principle. J. Math. Anal. Appl. 2015, 421, 806-829. [CrossRef]

13. Goyal, S.P.; Laddha, R.K. On the generalized Riemann zeta functions and the generalized Lambert transform. Ganita Sandesh 1997, 11, 99-108.

14. Jang, G.-W.; Kwon, H.-I.; Kim, T. A note on degenerate Apostol-Bernoulli numbers and polynomials. Adv. Stud. Contemp. Math. (Kyungshang) 2017, 27, 279-288.

15. Kim, T. An identity of the symmetry for the Frobenius-Euler polynomials associated with the fermionic $p$-adic invariant $q$-integrals on $Z_{p}$. Rocky Mt. J. Math. 2011, 41, 239-247. [CrossRef]

16. Kim, T. Identities involving Frobenius-Euler polynomials arising from non-linear differential equations. J. Number Theory 2012, 132, 2854-2865. [CrossRef]

17. Kim, D.S.; Kim, T. Some new identities of Frobenius-Euler numbers and polynomials. J. Inequal. Appl. 2012, 307, 1-10. [CrossRef]

18. Kim, D.S.; Kim, T. Higher-order Frobenius-Euler and poly-Bernoulli mixed-type polynomials. Adv. Differ. Equ. 2013, 251, 13. [CrossRef]

19. Kim, T.; Kim, D.S. Identities for degenerate Bernoulli polynomials and Korobov polynomials of the first kind. Sci. China Math. 2018. [CrossRef]

20. Kim, T.; Kim, D.S. An identity of symmetry for the degenerate Frobenius-Euler polynomials. Math. Slov. 2018, 68, 239-243. [CrossRef]

21. Kim, T.; Kwon, H.-I.; Seo, J.J. Some identities of degenerate Frobenius-Euler polynomials and numbers. Proc. Jangjeon Math. Soc. 2016, 19, 157-163.

22. Kim, T.; Mansour, T. Umbral calculus associated with Frobenius-type Eulerian polynomials. Russ. J. Math. Phys. 2014, 21, 484-493. [CrossRef]

23. Kurt, V. Some symmetry identities for the Apostol-type polynomials related to multiple alternating sums. Adv. Differ. Equ. 2013, 32, 1-32. [CrossRef]

24. Bayad, A.; Kim, T. Identities for Apostol-type Frobenius-Euler polynomials resulting from the study of a nonlinear operator. Russ. J. Math. Phys. 2016, 23, 164-171. [CrossRef]

25. Duran, U.; Acikgoz, M.; Araci, S. Hermite based poly-Bernoulli polynomials with a q-parameter. Adv. Stud. Contemp. Math. (Kyungshang) 2018, 28, 285-296.

26. Yang, S.L. An identity of symmetry for the Bernoulli polynomials. Discrete Math. 2008, 308, 550-554. [CrossRef]

27. Zhang, Z.; Yang, H. Several identities for the generalized Apostol-Bernoulli polynomials. Comput. Math. Appl. 2008, 56, 2993-2999. [CrossRef]

28. Milnor, J.W. Topology from the Differentiable View Point; University of Virginia Press: Charlottesville, VA, USA, 1965.

29. Yilmaz, B.; Özarslan, M.A. Frobenius-Euler and Frobenius-Genocchi polynomials and their differential equations. New Trends Math. Sci. 2015, 3, 172-180.

30. Marin, M.; Florea, O. On temporal behaviour of solutions in thermoelasticity of porous micropolar bodies. An. St. Univ. Ovidius Constanta-Ser. Math. 2014, 22, 169-188. [CrossRef]

31. Marin, M. Weak solutions in elasticity of dipolar porous materials. Math. Probl. Eng. 2008, $2008,158908$. [CrossRef]

(C) 2018 by the authors. Licensee MDPI, Basel, Switzerland. This article is an open access article distributed under the terms and conditions of the Creative Commons Attribution (CC BY) license (http://creativecommons.org/licenses/by/4.0/). 